Trauma Surgery \& Acute Care Open

\title{
Introducing high-flow nasal cannula oxygen therapy at the intermediate care unit: expanding the range of supportive pulmonary care
}

\author{
Joost D J Plate, ${ }^{1}$ Luke P H Leenen, ${ }^{1}$ Marc Platenkamp, ${ }^{2}$ Joost Meijer, ${ }^{3}$ Falco Hietbrink'
}

\begin{abstract}
'Division of Surgery, Universitair Medisch Centrum Utrecht, Utrecht University, Utrecht, The Netherlands

2Department of Intensive Care Medicine, University Medical Centre Utrecht, Utrecht University, Utrecht, The Netherlands

${ }^{3}$ Department of of Intensive Care Medicine, Noordwest Ziekenhuisgroep, Utrecht, The Netherlands
\end{abstract}

Correspondence to Joost D J Plate, Division of Surgery, Universitair Medisch Centrum Utrecht, Utrecht, 3584CX, The Netherlands; j.d.j.

Received 8 March 2018 Revised 4 April 2018 Accepted 18 April 2018 plate@umcutrecht.nl

\section{ABSTRACT}

Background Non-invasive respiratory support is a frequent indication for intermediate care unit (IMCU) admission. Extending the possibilities of respiratory support at the IMCU with high-flow nasal cannula oxygen therapy (HFNC) may prevent intensive care unit (ICU) transfer and invasive ventilation. However, the safety and limitations of HFNC administration in the stand-alone IMCU setting are not yet studied. This study therefore aims to investigate to what extent and in which patients HFNC can safely be administered at a stand-alone mixed surgical IMCU.

Methods A case series, using retrospectively collected data, was performed after the first year of introducing HFNC at a stand-alone IMCU. The following variables were collected: indication to start HFNC, vital parameters and arterial blood gas measurements. Primary outcome was 30-day mortality. Secondary outcome was transfer to the ICU.

Results A total of 96 admissions were included. The indications to start HFNC at the IMCU were predominantly pathologies of pulmonary origin $(n=67$, $69.8 \%$ ). Less frequent indications were prolonged support postweaning $(n=15)$, non-pulmonary sepsis $(n=7)$ and post-trauma resuscitation $(n=6)$. The average $\mathrm{PaO}_{2} / \mathrm{FiO}_{2}$ ratio at start of HFNC was $152(95 \% \mathrm{Cl} 139$ to 165). 30-day mortality was 7 , of which 5 were admitted with treatment restrictions (no ICU policy) and 2 deaths were unrelated to HFNC. Transfer to the ICU occurred in $18(18.8 \%)$ admissions, of which 12 received invasive mechanical ventilation. Reason for ICU transfer was mainly $\mathrm{PaO} 2 / \mathrm{FiO} 2$ ratio $<100$ under maximum noninvasive treatment $(n=12,66.7 \%)$. Application of HFNC at the IMCU prevented 162 days of ICU admission. Discussion Administration of HFNC at a stand-alone surgical IMCU may be safe as it expands the range of supportive possibilities and thereby reduces the need for ICU admissions. Pulmonary indications to start HFNC increase the risk of ICU transfer and mechanical ventilation.

Level of evidence Level VI.

\section{INTRODUCTION}

employer(s)) 2018. Re-use permitted under CC BY-NC. No commercial re-use. See rights and permissions. Published by BMJ.

To cite: Plate JDJ, Leenen LPH, Platenkamp M, et al. Trauma Surg Acute Care Open 2018;3:e000179.
Non-invasive respiratory support is a common reason to admit patients to the intermediate care unit (IMCU). Through extending the possibilities of the respiratory capabilities at the IMCU, intensive care unit (ICU) admission and mechanical ventilation may be prevented.

Mechanical ventilation is almost always a limitation of IMCUs, but an increase in the range of other supportive respiratory care at IMCUs can reduce the need to transfer patients from the IMCU to the ICU. ${ }^{1}$ In an IMCU in which the admitting specialist remains in charge (a so-called 'open' format), this reduces handovers to the intensivist. In this case, the admitting specialist continues to provide specialized care. ${ }^{2}$ Whether extended possibilities can safely be applied at a (stand-alone) IMCU and for which patients, however, is not yet studied.

All IMCUs provide standard respiratory support, which includes the administration of oxygen via nasal cannula, nasal tube or (non-rebreathing) oxygen mask. ${ }^{1}$ Extended respiratory support, which entails high-flow nasal cannula oxygen therapy (HFNC), non-invasive mechanical ventilation (NIV) and invasive mechanical ventilation, however, is less common. Especially the administration of HFNC at IMCUs is uncommon, as this was only reported as a possibility at one IMCU. ${ }^{13}$

HFNC is an oxygen administration method which allows for delivering warmed and humidified gas (air and oxygen) at very high flow rates via a nasal cannula. This improves the oxygenation, decreases respiratory work and improves patients' well-being and is a useful option in patients with acute hypoxic respiratory failure. ${ }^{4}$ In addition, it has shown to significantly decrease mortality and intubation rates compared with standard oxygen therapy, potentially making this technique a suitable replacement for standard respiratory support. ${ }^{5} 6$ Also, HFNC can be used for acute respiratory failure when the standard methods of respiratory support are insufficient and NIV or intubation are not (yet) indicated. ${ }^{7}$ However, hypercapnic respiratory failure is a limitation of HFNC administration.

HFNC was recently implemented in our standalone mixed surgical IMCU. The aim was to assess the safety of HFNC provided at the IMCU.

\section{METHODS}

\section{Study design and setting}

A case series, using retrospectively collected data, was conducted at the surgical IMCU of the University Medical Centre in Utrecht, a tertiary university referral hospital in the Netherlands. This independent, surgical IMCU admits patients from all surgical disciplines, providing hemodynamic monitoring and cardiovascular and respiratory support including inotropic use and supplementary oxygen. It has an open format with 24/7 supervision of surgeons with additional critical care certifications for hemodynamic and respiratory 
support. Its limitations are non-invasive and invasive mechanical ventilation, continuous renal replacement therapy and-until recently-HFNC. A general mixed-specialty closed format ICU run by intensivists is available for consultation and take over if necessary. In case of maximum HFNC support, intubation at the IMCU is required before patient transfer since transport with HFNC is logistically not possible in our setting.

In July 2016, HFNC was implemented at the IMCU to provide the possibility for extended respiratory support in patients with non-hypercapnic hypoxemic respiratory failure. The medical criterion to start HFNC was inadequate oxygenation under supportive respiratory care with an oxygen mask at 40\%-100\% oxygen, $10-15 \mathrm{~L} / \mathrm{min}$. To wean from HFNC, the protocol was to first lower the $\mathrm{FiO}_{2}$ and then the oxygen flow. Subsequently, at HFNC settings of $30 \% \mathrm{FiO}_{2}$ with $30 \mathrm{~L} / \mathrm{min}$, a switch to the (non-rebreathing) oxygen mask was considered. These settings were lowered after assessment of the arterial blood gas and pulse oximetry measurements at the discretion of the physician (in collaboration with the nurses). A respiratory therapist was not involved in this weaning process.

After training of the nurses and doctors with the equipment, the first patient received HFNC at 15 July 2016. From that date until 16 August 2017, all patients which received HFNC during IMCU admission were included in this study.

According to the Institutional Review Board, the study was not subject to the Medical Research Involving Human Subjects Act and therefore the necessity of informed consent was waived.

\section{Study variables: baseline}

The following baseline variables were collected: age, sex, admission location, underlying diagnosis of hospital admission, the admission duration at the IMCU, cardiovascular and pulmonary comorbidities, the Sequential Organ Failure Assessment (SOFA) score $^{8}$ and the American Society of Anesthesiologists (ASA) classification. ${ }^{9}$ If an item of the SOFA score was not measured within 3 days before admission at the IMCU, this item was considered normal. This approach assumes that there was-apparentlyno clinical reason to measure this and thus abnormalities are unlikely.

\section{Study variables: indication HFNC and vital signs}

To explore the indication of HFNC and the actual patient condition at the start of HFNC, the following variables were collected: indication to start (categorized in four categories: pathologies of pulmonary origin, postweaning of mechanical ventilation (ICU or postanesthesia care unit), sepsis (due to non-pulmonary causes) and post-trauma resuscitation), vital parameters (heart rate, mean arterial pressure, respiratory rate, saturation), laboratory investigations (CRP and leukocyte count) and an arterial blood gas within 6 hours of start of the HFNC, including a calculation of the $\mathrm{P} / \mathrm{F}$ ratio $\left(\mathrm{PaO}_{2}\right.$ of oxygen $\left(\mathrm{PaO}_{2}\right) /$ fraction of inspired oxygen $\left(\mathrm{FiO}_{2}\right)$ ratio).

The administered $\mathrm{FiO}_{2}$ of patients that received oxygen via nasal cannula or tube before receiving HFNC, was calculated according to previous measurements concerning intratracheal $\mathrm{FiO}_{2}$ for 2 and $4 \mathrm{~L}$ of oxygen per minute. ${ }^{10}$ The $\mathrm{FiO}_{2}$ values for 3,5 and $6 \mathrm{~L}$ of oxygen administration per minute were, respectively, intrapolated and extrapolated using their provided formula. This has led to an approximated $\mathrm{FiO}_{2}$ of $29.6 \%$ in $2 \mathrm{~L} /$ $\min , 32.75 \%$ for $3 \mathrm{~L} / \mathrm{min}, 35.9 \%$ for $4 \mathrm{~L} / \mathrm{min}, 39.7 \%$ for $5 \mathrm{~L} / \mathrm{min}$ and $43.5 \%$ for $6 \mathrm{~L} / \mathrm{min}$. These values were based on a respiratory rate of 15 per minute, in healthy subjects. However, it is known that the $\mathrm{FiO}_{2}$ alveolar value drops in ill patients. In addition, low flow oxygen by cannula depends on breathing pattern. ${ }^{11}$ To determine the $\mathrm{P} / \mathrm{F}$ ratio at the time of ICU transfer (if applicable), the set $\mathrm{FiO}_{2}$ at the HFNC was used.

\section{Study variables: utilization of HFNC}

The utilization of HFNC was analyzed using the following variables: flow (in $\mathrm{L} / \mathrm{min}$ ) and $\mathrm{FiO}_{2}$ (in percentage) at start, duration of the HFNC at the IMCU, maximal flow (in L/min) and maximal $\mathrm{FiO}_{2}$ (in percentage) during HFNC support. Whether the ICU was consulted at the start or during HFNC administration was also reported.

\section{Study outcome: mortality and ICU transfer}

To assess the safety of HFNC at the IMCU, the 30-day mortality after the start of HFNC was used as the primary outcome and transfer to the ICU rate as secondary outcome. In our hospital, HFNC was also occasionally used as maximal conservative treatment in the (elderly) patient without chances of recovery and non-ICU transfer policy. The overall 30-day mortality was subdivided into deaths with and without restrictions to maximal treatment.

The secondary outcome parameter, ICU transfer rate, was complemented with both the arterial blood gas at transfer and the reason for ICU transfer (intubation, NIV or other reasons). Transfers from the IMCU via the operating room to the ICU were not seen as a negative (or undesired) outcome, since these patients had an underlying problem which needed surgical therapy and thus were not transferred to the ICU for increased respiratory support. Hence, HFNC administration at the IMCU was classified as successful as the patient was subsequently transferred to the hospital ward or operating room and unsuccessful if a patient was transferred to the ICU.

\section{Statistical analyses}

Continuous variables were described with the mean and the $95 \%$ bias-corrected and accelerated CIs, to also describe the skewness of the data. To analyze the associations between variables, the following univariable analyses were performed: Kruskal-Wallis tests (continuous outcomes) and the Fisher's exact test (categorical variables). In comparing the successful versus unsuccessful administrations of HFNC, deaths at the IMCU were excluded since these patients were admitted for another reason (maximum supportive care) and hence, appropriateness of HFNC admissions could not be determined. A multivariable logistic regression analysis to identify predictors for ICU transfer was not performed due to too few events.

Throughout the analyses, a level of significance of 0.05 was used. All statistical analyses were performed using $\mathrm{R}$ software for statistical computing V.3.3.2, ${ }^{12}$ with the additional package 'bootstrap'. ${ }^{13}$

\section{RESULTS}

An overview of the baseline characteristics of admissions at the IMCU during the study period is provided in table 1 . A total of 96 admissions were included.

\section{Indications to start HFNC}

The indications for HFNC were predominantly due to pulmonary pathologies $(n=68,70.8 \%)$ (table 2$)$. Postweaning $(n=15)$, non-pulmonary sepsis $(n=7)$ and post-trauma resuscitation $(n=6)$ were less frequent indications to start HFNC at the IMCU. Of the patients with atelectasis, four also had pleural effusions due to side effects from fasciitis, pancreatitis or vascular disease. 
Table 1 Baseline characteristics of admissions for high-flow nasal cannula oxygen therapy

\begin{tabular}{lc}
\hline & Total, $\mathrm{n}=96(\%)$ \\
\hline Sex, male (\%) & $70(72.92 \%)$ \\
\hline Age, mean (95\% Cl) & $61.9(\mathrm{Cl} 58.3$ to 65.4$)$ \\
\hline Admission location, $\mathrm{n}(\%)$ & \\
\hline Emergency room & $16(16.7 \%)$ \\
\hline Intensive care unit & $27(28.1 \%)$ \\
\hline Other hospital & $4(4.2 \%)$ \\
\hline Recovery unit & $10(10.4 \%)$ \\
\hline Hospital ward & $39(40.6 \%)$ \\
\hline Underlying diagnoses, $\mathrm{n}$ (\%) & \\
\hline Trauma & $36(37.5 \%)$ \\
\hline Postoperative abdominal surgery & $26(27.1 \%)$ \\
\hline Esophagogastric bypass surgery & $15(15.6 \%)$ \\
\hline Extra-abdominal surgery & $8(8.3 \%)$ \\
\hline Severe necrotizing tissue disease & $3(3.11 \%)$ \\
\hline Exchange bed & $2(2.1 \%)$ \\
\hline Other & $6(6.3 \%)$ \\
\hline Admission duration in hours, mean (BCA 95\% Cl) & $98.8(78.9$ to 118.7$)$ \\
\hline Comorbidity, $n$ (\%) & \\
\hline Cardiovascular & $50(52.1 \%)$ \\
\hline Pulmonary (COPD/asthma) & $8(8.3 \%)$ \\
\hline Pulmonary (other) & $4(4.2 \%)$ \\
\hline SOFA score, mean (BCA 95\% Cl) & $3.7(3.3$ to 4.1$)$ \\
\hline ASA classification, $\mathrm{n}$ (\%) & $27(28.1 \%)$ \\
\hline I & $30(31.3 \%)$ \\
\hline III & $37(38.5 \%)$ \\
\hline IV & $2(2.1 \%)$ \\
\hline
\end{tabular}

This table shows the baseline characteristics of all admissions at the intermediate care unit, which received high-flow nasal cannula oxygen therapy from 15 July 2016 to 16 August 2017

ASA, American Society of Anesthesiologists; BCA, bootstrapped confidence interval, COPD, chronic obstructive pulmonary disease; SOFA, Sequential Organ Failure Assessment.

The pleural effusion in the fasciitis and abdominal aortic aneurism patients were presumed reactive and therefore drained, after which the respiratory status improved and patients were weaned from HFNC within a couple of hours. In the vascular patients, the pleural effusion was supposed to reflect a (partial) fluid overload in patients with decreased cardiac function. No draining was performed in these patients.

\section{Vital signs at the start of HFNC}

Table 3 shows the vital signs at the start of HFNC at the IMCU. There were no significant differences in vital signs per indication.

\section{Utilization of HFNC at the IMCU}

On average, the starting values of the HFNC at the IMCU were $38 \mathrm{~L} / \mathrm{min}$ flow (95\% CI 36 to 40) and 59\% $\mathrm{FiO}_{2}(95 \% \mathrm{CI}$ 57.0 to 61.5 ). The average duration of administered HFNC was 40.4 hours (95\% CI 33.5 to 49.3 ). Maximum settings used during IMCU admission were on average 42 (95\% CI 40 to 43 ) $\mathrm{L} / \mathrm{min}$ and $68 \%$ (95\% CI 65 to 71 ) $\mathrm{FiO}_{2}$. There were no (significant) differences in utilization per indication or underlying diagnoses. In total, HFNC was administered for 3878 hours (162
Table 2 Indications to start high-flow nasal cannula oxygen therapy

\begin{tabular}{lc}
\hline & Total, $\mathrm{n}=96(\%)$ \\
\hline Pulmonary & $67(69.8 \%)$ \\
\hline Pneumonia & $21(21.9 \%)$ \\
\hline Atelectasis with pleural fluid & $11(11.5 \%)$ \\
\hline Combination of pulmonary causes & $10(10.4 \%)$ \\
\hline Fluid overload & $10(10.4 \%)$ \\
\hline ARDS & $1(1.0 \%)$ \\
\hline Pneumothorax & $4(4.2 \%)$ \\
\hline Sputum stasis & $3(3.1 \%)$ \\
\hline Pulmonary embolism & $3(3.1 \%)$ \\
\hline Aspiration pneumonia & $2(2.1 \%)$ \\
\hline Morphine intoxication & $1(1.0 \%)$ \\
\hline Postweaning & $15(15.6 \%)$ \\
\hline From recovery unit & $12(12.5 \%)$ \\
\hline From ICU & $3(3.1 \%)$ \\
\hline Sepsis (non-pulmonary) & $7(7.3 \%)$ \\
\hline Post-trauma resuscitation & $7(7.3 \%)$ \\
\hline
\end{tabular}

This table shows the indications to start high-flow nasal cannula oxygen therapy at the intermediate care unit.

ARDS, acute respiratory distress syndrome; ICU, intensive care unit.

days) during the study period. The intensivist was consulted in $41(42.7 \%)$ admissions.

\section{Mortality}

The 30-day mortality was seven (table 4). Of these deaths, five were admitted at the IMCU with treatment restrictions (policy at admission was to not admit at the ICU anymore, due to metastases $(n=2)$ or bad general condition $(n=3))$. Of the two deaths without treatment restrictions, one died at the hospital ward 5 days after the end of HFNC treatment at the IMCU; this patient died of an intra-abdominal bleeding with unknown cause. The other was discharged home in good medical condition, although

\begin{tabular}{lcc}
$\begin{array}{l}\text { Table } 3 \\
\text { therapy }\end{array}$ & Vital signs at the start of high-flow nasal cannula oxygen \\
\hline & Mean (BCA $95 \%$ Cl) & Missing, $\mathbf{n}(\%)$ \\
\hline Heart rate & $95(91$ to 99$)$ & $10(10.4 \%)$ \\
\hline Mean arterial pressure & $92.90(89.42$ to 96.53$)$ & $10(10.4 \%)$ \\
\hline Respiratory rate & $20(19$ to 22$)$ & $12(12.5 \%)$ \\
$\mathrm{SpO}_{2}$ & $94(93$ to 94$)$ & $10(10.4 \%)$ \\
$\mathrm{pH}$ & $7.41(7.40$ to 7.43$)$ & $24(25.0 \%)$ \\
$\mathrm{pCO}_{2}$ & $39.6(38.2$ to 41.1$)$ & $24(25.0 \%)$ \\
$\mathrm{pO}_{2}$ & $72.7(69.4$ to 76.8$)$ & $24(25.0 \%)$ \\
$\mathrm{HCO}_{3}$ & $24.8(23.9$ to 25.6$)$ & $24(25.0 \%)$ \\
$\mathrm{BE}_{\mathrm{Std}} \mathrm{HCO}{ }_{3}$ & $0.12(-0.90$ to 1.04$)$ & $24(25.0 \%)$ \\
\hline Saturation & $24.8(24.0$ to 25.5$)$ & $28(29.2 \%)$ \\
\hline $\mathrm{P} / \mathrm{F}$ ratio & $94 \%(93$ to 94$)$ & $25(26.0 \%)$ \\
CRP & $152.7(139.8$ to 166.0$)$ & $28(29.2 \%)$ \\
Leukocyte count & $163(144$ to 185$)$ & $9(9.4 \%)$ \\
\hline
\end{tabular}

This table shows the vital parameters, arterial blood gas values and laboratory investigations just before the start of high-flow nasal cannula oxygen therapy at the intermediate care unit.

$\mathrm{CRP}, \mathrm{C}$ reactive protein; $\mathrm{BE}$, base excess; $\mathrm{P} / \mathrm{F}, \mathrm{PaO}_{2} / \mathrm{FiO}_{2}$ 


\begin{tabular}{|c|c|}
\hline & Total, $\mathrm{n}=96$ \\
\hline 30-day mortality & $7(7.3 \%)$ \\
\hline Treatment restrictions* & $5(5.2 \%)$ \\
\hline No treatment restrictions & $2(2.1 \%)$ \\
\hline Transfer to the ICU, total & $24(25.0 \%)$ \\
\hline Via operation room & $6(6.3 \%)$ \\
\hline Directly, for & $18(18.8 \%)$ \\
\hline Intubation & $12(12.5 \%)$ \\
\hline NIV & $5(5.2 \%)$ \\
\hline Continuation high-flow oxygen (+RRT) & $1(1.0 \%)$ \\
\hline
\end{tabular}

This table shows the mortality and transfer to the ICU rate in the studied population. It shows the 30-day mortality as well as transfer to the ICU rate and indications for this ICU transfer.

* Oncologic (metastatic) patients or admissions for maximal supportive care at the IMCU (a no-ICU policy at admission).

ICU, intensive care unit; IMCU, intermediate care unit; NIV, non-invasive ventilation; RRT, renal replacement therapy.

with the wish for euthanasia. The eventual cause of death was unknown.

\section{Transfer to the ICU}

A total of $18(18.8 \%)$ admissions were transferred to the ICU. The reason for ICU transfer was a low $\mathrm{P} / \mathrm{F}$ ratio (mean 75.3 (95\% CI 67.19 to 83.15), all below 100) in 12 admissions, hypercapnia in 3 admissions, shock in 1, intolerance for HFNC in 1 , renal replacement therapy in 1 and clinical manifestations of respiratory muscle failure in 1 admission. Mean time from start HFNC to ICU transfer was 38.32 hours (95\% CI 26.05 to 54.89)

Of these 18 ICU transfers, $12(66.7 \%)$ received invasive mechanical ventilation, 5 received non-invasive mechanical ventilation and 1 was transferred for renal replacement therapy while continuing HFNC. The 30-day mortality of ICU transfers was 0 .

In total, of the IMCU population that received HFNC, 12.5\% required invasive mechanical ventilation. Of the patients which were transferred to the ICU for invasive mechanical ventilation, $12(70.6 \%)$ were immediately transferred after initial intensivist consultation, while 5 were transferred after potential delay between ICU consultation and transfer.

The studied variables at admission, distinguished by those in who HFNC was successfully (transferred to ward or operating room) or unsuccessfully (transferred to ICU) administered are shown in table 5. Of all the unsuccessful cases, 10 (55.6\%) were initially admitted from the hospital ward, 4 from the emergency room, 3 from the ICU and 1 from the postanesthesia care unit.

Unsuccessful admissions were nearly always ( $\mathrm{n}=16,88.9 \%$ ) admissions which received HFNC for pulmonary indications. Per pulmonary indication to start HFNC, five admissions (38.6\%) with atelectasis and pleural fluid, six admissions with pneumonia and three with a combined pulmonary problem were transferred to the ICU. This did not occur in postweaning or sepsis (due to non-pulmonary indications) admissions. Of the post-trauma resuscitation admissions, three admissions (all with pulmonary contusion) were transferred to the ICU.

\section{DISCUSSION}

This study is the first to report to what extent and for which patients HFNC can safely be applied at the IMCU. The observed 30-day mortality was seven (7.3\%). However, these included five admissions with treatment restrictions at admission, while the other deaths were not related to the HFNC treatment. Transfers to the ICU $(18.8 \%)$ occurred mainly in case HFNC was initiated for pulmonary indications or for post-traumatic pulmonary contusion. Patients with atelectasis with pleural fluid, pulmonary contusion or pneumonia are at highest risk for ICU transfer. Transferred patients $(94.5 \%)$ received (non)-invasive mechanical ventilation at the ICU, most commonly due to low $\mathrm{P} / \mathrm{F}$ ratio $(<100)$ under maximum HFNC settings.

These findings indicate that the range of supportive respiratory care at IMCUs may safely be expanded with HFNC, provided there is sufficient knowledge and adequate triage. Since these patients were otherwise cared for on the ICU, this could savecostly-ICU capacity for the more severe patients (in the present study $>160$ ICU days). However, the possibility of HFNC at the IMCU could also have decreased the threshold for its administration. Although this perhaps means that not every HFNC patient at the IMCU would have been admitted at the ICU, it may still be preferable to the oxygen mask, as this is reported to significantly decrease the in-hospital mortality and need of mechanical ventilation as compared with conventional oxygen therapy. ${ }^{56}$ On the other hand, the low mean observed $\mathrm{P} / \mathrm{F}$ ratio of 152 (95\% CI 139.8 to 166.0$)$ indicates that most patients are at the more severe end of the spectrum of lung function limitation before the start of HFNC, ${ }^{14}$ contradicting a decreased threshold for the start of HFNC.

This study also provides a few tools for the physician to recognize those patients at risk for ICU transfer and mechanical ventilation, namely those patients with atelectasis with pleural fluid and pneumonia. For those patients, timely consultation of an intensivist should be considered. However, to truly adequately recognize patients at risk for ICU transfer, future research should focus on multivariable prediction of this outcome.

Earlier observational research toward the safety of administration of HFNC has been performed at the ICU. One study $(n=38)$ showed that-in patients with a mean PF ratio of 102 (SD of 23) - the ICU mortality was $7.9 \%$ with an invasive mechanical ventilation rate of $23.7 \% .{ }^{15}$ In another, randomised controlled trial $(n=106)$ in HFNC patients with an $\mathrm{PaO}_{2} / \mathrm{FiO}_{2}$ of 157 (SD of 89 ), the ICU mortality was $11 \%$ and 90 -day mortality was $12 \%$. The observed invasive mechanical ventilation rate was $38 \% .^{5}$ This present study is in line with these previous ICU reports in the context of a similar 30-day mortality. Althoughas discussed before-our IMCU included patients with restrictions to treatment (especially restrictions in ICU admission). On the other hand, our case-mix was likely different from that of the ICU in that the IMCU admitted mainly single organ failure patients, as indicated by the relatively low SOFA score of 3.7 (95\% CI 3.3 to 4.1 ). This underlines the importance of adequate triage.

Furthermore, since deteriorating HFNC patients frequently require mechanical ventilation, it is a necessity to have (rapid) backup from an adequately functioning ICU. Especially since HFNC in a stand-alone IMCU potentially delays ICU transfer, with subsequent acute need for mechanical ventilation and delay in deposition.

This study did not assess the safety of HFNC administration by comparing its use at the IMCU versus the ICU setting. However, we believe that the absence of any HFNC-related 
Table 5 Successful administration of high-flow oxygen therapy at the intermediate care unit.

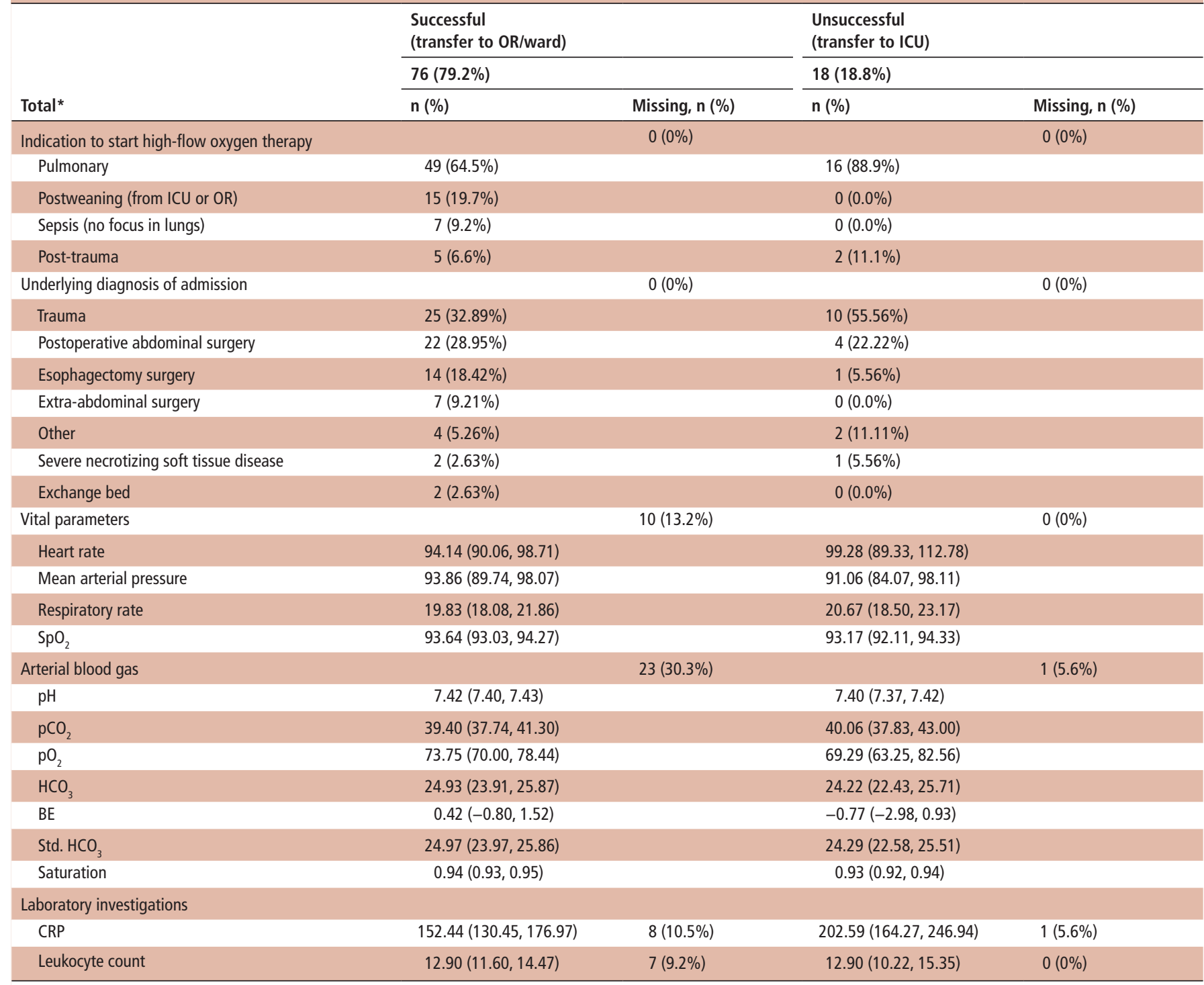

This table shows the indication to start high-flow nasal cannula oxygen therapy, underlying diagnoses, vital parameters and arterial blood gas measures for both patients for whom high-flow nasal cannula oxygen therapy was successfully and unsuccessfully administered.

Percentages are shown per column and $\mathrm{Cls}$ are $95 \%$ bias-corrected and accelerated $\mathrm{Cls}$.

${ }^{*}$ The total does not add up to $100 \%$, since there were two deaths at the IMCU, which were excluded from these analyses.

$\mathrm{CRP}, \mathrm{C}$ reactive protein; IMCU, intermediate care unit; $\mathrm{OR}$, operating room.

(30-day) mortality in these 96 patients indicates that administration of HFNC in this setting may be safe for non-hypercapnic hypoxia patients.

Since our low numbers of events hampered a valid-multivariable-identification of HFNC patients at risk for ICU transfer (and mechanical ventilation), this should also be the focus of further research. With the added knowledge that IMCU may be a safe setting to administer HFNC, an important first step can be taken in the process of acquiring this knowledge. Furthermore, in-depth analysis for specific subgroups of patients (eg, thoracic trauma or acute pancreatitis) was not possible due to the heterogeneous population of included patients, which reflects common practice at our IMCU.

\section{CONCLUSIONS}

The application of HFNC at a stand-alone surgical mixed IMCU may be safe and expands the range of respiratory support possibilities, reducing the need for ICU admissions. Pulmonary indications to start HFNC (especially atelectasis with pleural fluid and pneumonia) increase the risk of ICU transfer and mechanical ventilation. Further research is needed to identify risk factors for this ICU transfer.

Contributors JDJP made substantial contributions to design, data collection, data analysis and the interpretation. He was the main author involved in drafting and finalizing the manuscript. LPHL was involved in the design and critically revised the manuscript. He has given final approval of this manuscript to be published. MP made substantial contributions to conception and design and critically revised the manuscript. He has given final approval of the version to be published. JM has critically revised the manuscript. He has given final approval of the version to be published. FH contributed to the design and actively participated in data collection, analysis and its interpretation. He was involved in drafting the manuscript and revising it critically.

Funding The authors have not declared a specific grant for this research from any funding agency in the public, commercial or not-for-profit sectors.

Competing interests None declared.

Patient consent Not required. 
Ethics approval Institutional review board of the University Medical Centre Utrecht (protocol number 17-326/C).

Provenance and peer review Not commissioned; externally peer reviewed.

Open access This is an open access article distributed in accordance with the Creative Commons Attribution Non Commercial (CC BY-NC 4.0) license, which permits others to distribute, remix, adapt, build upon this work non-commercially, and license their derivative works on different terms, provided the original work is properly cited, appropriate credit is given, any changes made indicated, and the use is non-commercial. See: http://creativecommons.org/licenses/by-nc/4.0/.

\section{REFERENCES}

1 Plate JDJ, Leenen LPH, Houwert M, Hietbrink F. Utilisation of Intermediate Care Units: A Systematic Review. Crit Care Res Pract 2017;2017:1-10.

2 Yoo EJ, Damaghi N, Shakespeare WG, Sherman MS. The effect of physician staffing model on patient outcomes in a medical progressive care unit. Journal of Critical Care 2015:191.

3 Pirret AM. Utilizing TISS to differentiate between intensive care and high-dependency patients and to identify nursing skill requirements. Intensive Crit Care Nurs 2002;18:19-26.

4 Masclans JR, Pérez-Terán P, Roca O. The role of high flow oxygen therapy in acute respiratory failure. Med Intensiva 2015;39:505-15.

5 Frat JP, Thille AW, Mercat A, Girault C, Ragot S, Perbet S, Prat G, Boulain T, Morawiec E, Cottereau A, Et al. High-flow oxygen through nasal cannula in acute hypoxemic respiratory failure. N Engl J Med 2015;372:2185-96.
6 Zhao H, Wang H, Sun F, Lyu S, An Y. High-flow nasal cannula oxygen therapy is superior to conventional oxygen therapy but not to noninvasive mechanica ventilation on intubation rate: a systematic review and meta-analysis. Crit Care 2017;21:184.

7 Gotera C, Díaz Lobato S, Pinto T, Winck JC. Clinical evidence on high flow oxygen therapy and active humidification in adults. Rev Port Pneumol 2013:19:217-27.

8 Jones AE, Trzeciak S, Kline JA. The Sequential Organ Failure Assessment score for predicting outcome in patients with severe sepsis and evidence of hypoperfusion at the time of emergency department presentation. Crit Care Med 2009;37:1649-54.

9 Saklad M. Grading of patients for surgical procedures. Anesthesiology 1941;2:281-4.

10 O'Reilly Nugent A, Kelly PT, Stanton J, Swanney MP, Graham B, Beckert L. Measurement of oxygen concentration delivered via nasal cannulae by tracheal sampling. Respirology 2014;19:538-43.

11 Wettstein RB, Shelledy DC, Peters Jl. Delivered oxygen concentrations using low-flow and high-flow nasal cannulas. Respir Care 2005;50:604-9.

12 Core Team R. R:A Language and Environment for Statistical Computing. Vienna, Austria: R Foundation for Statistical Computing, 2017

13 Soriginal and from StatLib and by Rob Tibshirani R port by Friedrich Leisch: Bootstrap: Functions for the Book "An Introduction to the Bootstrap", 2017. R package version.

14 Bernard GR, Artigas A, Brigham KL, Carlet J, Falke K, Hudson L, Lamy M, Legall $J R$, Morris A, Spragg R. The American-European Consensus Conference on ARDS. Definitions, mechanisms, relevant outcomes, and clinical trial coordination. Am J Respir Crit Care Med 1994;149:818-24.

15 Sztrymf B, Messika J, Bertrand F, Hurel D, Leon R, Dreyfuss D, Ricard JD. Beneficial effects of humidified high flow nasal oxygen in critical care patients: a prospective pilot study. Intensive Care Med 2011;37:1780-6. 